Psicooncología

ISSN: $1696-7240$

http://dx.doi.org/10.5209/PSIC.57092

\title{
Tratamiento psicológico de la emesis anticipatoria
}

Autora: Laura Haro Martínez

Tipo de trabajo: tesis doctoral

Dirección: Juan Antonio Cruzado

Universidad: Universidad Complutense de Madrid

Fecha de lectura: 21 de junio de 2017

E-mail: laura.haro@aecc.es

\section{Resumen}

Introducción La emesis por quimioterapia sigue siendo uno de los principales problemas que presentan los pacientes oncológicos y que puede afectar significativamente a su calidad de vida. La emesis anticipatoria es una respuesta aprendida a la quimioterapia que, según los estudios de Morrow, Roscoe, Kirshner, Hynes, y Rosenbluth en 1998, ocurre en un 25 \% de los pacientes sobre el cuarto ciclo de tratamiento. Es una respuesta condicionada que aparece en pacientes que han desarrollado náuseas y vómitos importantes durante los ciclos previos de quimioterapia. Roscoe, Morrow, Aapro, Molassiotis y Olver en 2011 señalaban que este tipo de emesis es difícil de controlar mediante fármacos. Existen dos factores que influyen en su aparición: clínicos o farmacológicos (potencial emético, método de administración, número y duración de las sesiones) y psicológicos (nivel de ansiedad previo a la quimioterapia o posibilidad de distracción durante la misma). La Guia NCCN Antiemesis 2015 resalta la importancia de la prevención en cada ciclo de tratamiento. Si aparece emesis anticipatoria el tratamiento se basaría en relajación, desensibilización sistemática, hipnosis e imaginación guiada junto con la musicoterapia, acupuntura y como tratamiento farmacológico se podrían usar benzodiacepinas que añadidas al tratamiento antiemético y al apoyo psicológico, parecen mejorar los vómitos anticipatorios (Razavi et al., 1993; Malik et al., 1995).

Objetivos: el objetivo principal es estudiar la eficacia de la relajación muscular progresiva para la prevención y el tratamiento de la emesis anticipatoria, ansiedad, depresión y calidad de vida en pacientes con cáncer de mama que van a recibir quimioterapia. Los objetivos específicos son valorar la eficacia del tratamiento de relajación para el control de la emesis, calidad de vida, ansiedad y depresión, y analizar los cambios en la calidad de vida de las pacientes viendo si ésta mejora tras dos años de finalizar la QT. La principal hipótesis formulada se basa en comprobar que los 
pacientes, del grupo tratamiento, que practican la técnica de Relajación Muscular tendrán menos emesis aguda, tardía y anticipatoria, en comparación con el grupo que no lleva a cabo ninguna intervención. Además tendrán menos síntomas de ansiedad y depresión, y mejores niveles de calidad de vida en comparación con el grupo no tratado.

Material y métodos: se incluyeron 46 pacientes diagnosticadas de cáncer de mama. El estudio tuvo una duración de cuatro años desde octubre de 2012 hasta julio de 2016. Las pacientes eran asignadas a dos grupos de forma aleatoria. Al grupo experimental se le enseñaba la técnica de relajación muscular progresiva. Se practicaba media hora antes de recibir el primer ciclo de quimioterapia y durante las tres semanas después una vez al día hasta el siguiente ciclo (el ejercicio se entregaba en un CD que el paciente se llevaba a casa), mientras que el grupo control no recibía ninguna instrucción previa. Todas las pacientes llevaban la misma medicación antiemética.

Todas las pacientes fueron evaluadas en 4 ocasiones. En la medida pretratamiento y postratamiento se usan los cuestionarios de calidad de vida (QLQ-C30 y BR-23) y el HAD para medir síntomas de ansiedad y depresión. Tras cada ciclo de QT, rellenaban el cuestionario MAT que mide emesis aguda y tardía. En el seguimiento sólo se evalúa la calidad de vida. Los criterios de inclusión fueron: sexo femenino, diagnóstico de cáncer de mama (estadios I, II y III), recibir quimioterapia Adriamicina/ Ciclofosfamida y consentimiento escrito. Se excluyeron las pacientes metastásicas, aquellas que sólo recibían hormonoterapia o radioterapia, las que no mostraran una capacidad cognitiva adecuada para responder el cuestionario o no entendieran bien el castellano, o las participantes que no cumplimentaban los cuestionarios.

Resultados: con respecto a las variables de ansiedad y depresión, las pacientes de ambos grupos presentan valores normales en estas dos variables tanto antes de empezar el tratamiento como al finalizarlo. Podemos resaltar que el grupo no tratado mejora en ansiedad. En cuanto a la calidad de vida, las pacientes de ambos grupos presentan buen funcionamiento emocional, cognitivo, social y en la escala de salud global. Encontramos un empeoramiento del funcionamiento físico y cognitivo en las pacientes del grupo no tratado y una mejoría de la función rol en el grupo que practica la técnica de relajación con el paso del tiempo. Los síntomas como la fatiga, náuseas, dolor, insomnio, apetito, diarrea y el aspecto económico no mostraron cambios durante todo el estudio, ni tampoco encontramos diferencias entre grupos. Encontramos diferencias significativas entre los dos grupos al terminar la QT, con respecto a la disnea y estreñimiento. Con respecto a las puntuaciones en el EORTC BR-23, las puntuaciones en imagen corporal empeoran en el grupo control $(p=0,024)$ del inicio al final del tratamiento. Encontramos una importante afectación del área sexual tanto en el funcionamiento como en el disfrute. En ambos grupos, el estado físico empeora tras la QT por los efectos secundarios de la misma $(\mathrm{p}=0,000)$. Los síntomas locales de la mama, el brazo, y la preocupación por la pérdida de pelo muestran una escasa afectación. En relación a la emesis, no encontramos diferencias importantes entre ambos grupos en los diferentes ciclos de tratamiento, sólo destacar que existen diferencias significativas entre los dos grupos tras la primera QT en la aparición de náuseas agudas $(\mathrm{p}=0,022)$ y también existen diferencias entre las náuseas tardías en los diferentes ciclos de tratamiento en el grupo experimental $(p=0,023)$.

Conclusiones: el tratamiento de relajación no previene la aparición de náuseas o vómitos. El tratamiento de relajación no disminuye los niveles de ansiedad y/o depresión de los pacientes. El tratamiento de relajación muscular no tiene repercusiones importantes en la calidad de vida de las pacientes con cáncer de mama. 